\title{
ALOCAÇÃO DE RECURSOS FINANCEIROS EM PROJETOS DE RISCO NA EXPLORAÇÃO DE PETRÓLEO
}

Francisco Nepomuceno Filho

Geofísico da Divisão de Exploração do Cenpes, Petrobras (Rio de Janeiro, Brasil).

E-mail: nepomuceno@braspetro.petrobras.com.br

Saul B. Suslick

Professor do Departamento de Administração e Política de Recursos Minerais, Instituto de Geociências, Unicamp (Campinas, São Paulo, Brasil).

E-mail: suslick@ige.unicamp.br

RESUMO

As recentes mudanças no setor petrolifero implicam um novo ambiente de exploração de intensa competição no Brasil. As tendências de internacionalização da exploração obrigam as empresas a adotarem técnicas padronizadas de avaliação $e$ comparação de prospectos visando à redução do risco. Dentro dessa perspectiva, os indivíduos necessitam de um processo decisório que contemple os objetivos da empresa, as metas desejadas e as restrições orçamentárias. Este artigo descreve um sistema teórico e prático de suporte à tomada de decisão baseada na teoria da utilidade e da análise da decisão, com o objetivo de estimar a melhor alocação de capital em uma bacia sedimentar. Além disso, esse sistema possibilita estimar o melhor nível de participação financeira de uma firma em um projeto de exploração de petróleo realizado em parceria.

\section{ABSTRACT}

With the new changes in the petroleum sector in Brazil, the exploration will enter in an increasingly competitive and risky business environment. The trends of internationalization of exploration and risk reducing recommend the use of standardized techniques of evaluation and comparison of plays and prospects. Under these circumstances, managers have a growing need to employ better and more systematic decisionprocesses that explicitly embody the firm's objectives, desired goals, and resource constraints. In order to spread risk in major projects, oil companies will continue to engage in joint ventures. This paper describes a theoretical and practical system for decision support based upon the expected utility theory and decision analysis, in order to estimate the best capital allocation and to define the best level of financial participation of a firm in the new ventures in petroleum exploration project.

PALAVRAS-CHAVE

Avaliação econômica de projetos, análise de risco, tomada de decisão, alocação de recursos, parcerias em exploração de petróleo.

KEY WORDS

Economic evaluation, risk analysis, decision making, budget allocation, petroleum exploration joint venture. 


\section{INTRODUÇÃO}

Com a quebra do monopólio do petróleo, entrou em vigor um novo programa de qualificação do orçamento de Exploração e Produção (E\&P) que, certamente, é bem diferente da distribuição histórica dos investimentos de exploração nas diversas bacias do Brasil.

O objetivo deste artigo é apresentar uma técnica que, nas fases de E\&P, permita comparar projetos de risco em diversas bacias, possibilitando a priorização de uma carteira de projetos, e identificar o nível ótimo de participação em cada projeto, auxiliando a decisão para futuras associações do tipo joint venture e/ou compra ou venda de participação em empreendimentos de petróleo.

Essa metodologia usa a teoria da utilidade para incorporar a avaliação econômica e a análise de risco no processo de tomada de decisão, levando em consideração a capacidade de investimento da firma e a disposição de seu gerente para correr riscos.

Este trabalho aborda o conceito do risco financeiro em exploração de petróleo, particularmente o risco associado com a perfuração de poços. $\mathrm{Na}$ exploração, o risco se relaciona com o potencial de perda, como o custo da perfuração de um poço seco versus a compensação de ganho com a descoberta e a produção de quantidades comerciais de petróleo, conforme mostra a Figura 1. Esse exemplo mostra a árvore de decisão de um projeto de perfuração de um poço com probabilidade de $25 \%$ de encontrar petróleo. Isso implica que, a cada quatro poços perfurados, obtém-se sucesso em um poço, com um ganho de $\mathrm{R} \$ 100$ milhões, enquanto com os três poços secos ocorrerá uma perda de $\mathrm{R} \$$ 15 milhões.

Na Figura 1, E, A, D e P são fases de investimentos de um projeto de $\mathrm{E} \& \mathrm{P}$ de petróleo $(\mathrm{E}=$ exploração, $\mathrm{A}=$ avaliação, $\mathrm{D}=$ desenvolvimento e $\mathrm{P}=$ produção) e VPL é o valor presente líquido do projeto.

Figura 1 - Fluxo de caixa de um projeto de perfuração de um poço de petróleo

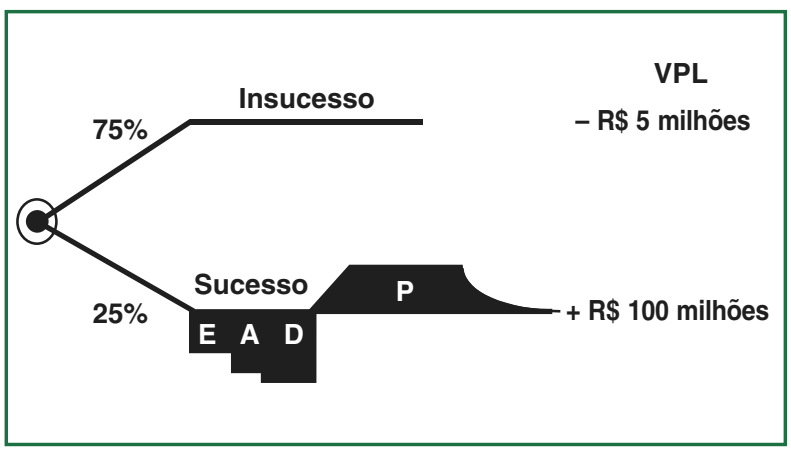

A partir desse exemplo, busca-se apresentar neste trabalho a visão de que o risco pode ser tratado sistematicamente pelo uso de teorias estatísticas e probabilísticas e que a preferência do gerente em relação ao risco pode ser expressa de modo quantitativo, dando consistência às decisões de alocação de capital nos projetos exploratórios.

\section{OS MÉTODOS TRADICIONAIS DE ALOCAÇÃO DE CAPITAL FREQÜENTEMENTE CONDUZEM A ESCOLHAS NÃO APROPRIADAS DE INVESTIMENTOS QUE APRESENTAM DIFERENTES RISCOS.}

A análise quantitativa do risco não elimina ou reduz o risco do projeto. É uma ferramenta para avaliar, quantificar e entender cada prospecto ${ }^{1}$ e seu risco associado, de forma que o gerente possa tomar melhores decisões estratégicas e financeiras. Após a identificação dos riscos associados a cada projeto, é possível compartilhar o risco financeiro do empreendimento via diversificação da carteira de projetos e via participação fracionada em cada projeto.

\section{FERRAMENTAS UTILIZADAS}

$\mathrm{Na}$ alocação de recursos financeiros em projetos de E\&P de petróleo, utiliza-se todo o instrumental da avaliação econômica de projetos que visa a determinar seu fluxo de caixa, sua taxa mínima de atratividade e seu valor presente líquido. Quando se dispõe da probabilidade de sucesso, calcula-se também o valor monetário esperado do projeto.

Além disso, neste trabalho aplica-se a teoria da utilidade, criada por Von Neumann e Morgenstern (1953), visando a definir a curva de preferência do gerente diante do risco, bem como seu nível de aversão ao risco diante da restrição do capital exploratório disponível.

Este trabalho faz parte da tese de douto- 
rado desenvolvida na Unicamp por Nepomuceno (1997), na qual foram criados alguns programas computacionais que sintetizam a teoria e facilitam a aplicação desse método em diversos contextos do processo exploratório.

\section{O MÉTODO DO VALOR MONETÁRIO ESPERADO}

Os métodos tradicionais de alocação de capital, tais como a análise do valor presente líquido (VPL), a taxa interna de retorno (TIR) e o valor monetário esperado (VME), freqüentemente conduzem a escolhas não apropriadas de investimentos que apresentam diferentes riscos. Esses métodos também fornecem pouca ou nenhuma visão sobre o valor e as alternativas para compartilhar riscos e diversificar os investimentos.

Muitos exploracionistas dizem que o conceito de valor monetário esperado (VME) inclui uma ponderação da conseqüência financeira pela sua probabilidade de sucesso. Entretanto, risco não é função somente da distribuição probabilística de resultados de reservas a alcançar, mas também da magnitude do capital sendo exposto à chance de perda.

Valor monetário esperado (VME) de um projeto é a soma do montante de capital ganho ou perdido se cada resultado ocorrer multiplicado pela sua probabilidade de ocorrência. Pode-se dizer que o VME é o caso do tudo ou nada. Ele não especifica o nível ótimo de participação no projeto, é indiferente ao risco de grandes perdas financeiras e assume que o capital da empresa é ilimitado.

Se o investimento representar uma pequena fração do orçamento da empresa, o decisor poderá conservar seu critério baseado no VME. Entretanto, se a possibilidade de perda for significativa, podendo abalar as finanças da empresa, seus critérios deverão ser revistos.

Nesse sentido, a noção de risco varia de uma empresa para outra em função de sua capacidade de absorver perdas. Um mesmo projeto pode ser considerado arriscado para uma firma e atrativo para outra. Nesse processo, três variáveis são importantes: a razão prêmio/custo do investimento, a razão capital da firma/custo do investimento e a proba- bilidade de sucesso do empreendimento, pontos que serão abordados mais adiante.

\section{MÉTODO DA TEORIA DA UTILIDADE}

O projeto de risco é inicialmente analisado pela técnica do seu valor monetário esperado, sendo necessário atribuir uma probabilidade a cada evento (Figura 2). A taxa de juros que anula o valor monetário esperado é definida como a taxa de rentabilidade interna do projeto, já levando em conta o risco.

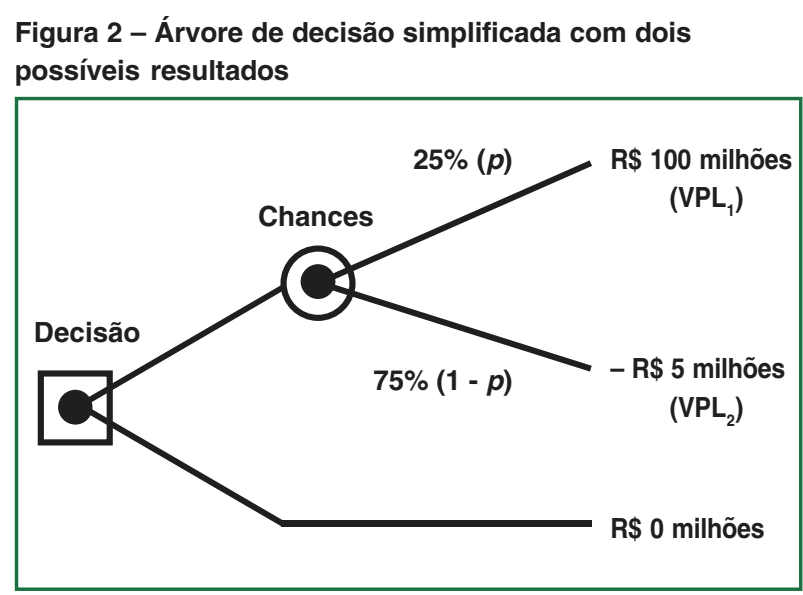

Na Figura 2, o quadrado representa uma decisão, o círculo representa as alternativas de resultados do projeto e $p$ é a probabilidade de sucesso do projeto. $\mathrm{O}$ valor monetário esperado calculado para o projeto da Figura 2 é dado pela Equação 1 e equivale a $\mathrm{R} \$ 21,25$ milhões.

$$
\mathrm{VME}=p \times \mathrm{VPL}_{1}+(1-p) \times \mathrm{VPL}_{2}
$$

(Equação 1)

\section{COMPORTAMENTO DIANTE DO RISCO}

Normalmente, os pessoas mostram aversão ao risco quando se aumenta o valor monetário do investimento $^{2}$ (Figura 3).

Na Figura 3, no eixo dos $x$, têm-se os valores monetários dos possíveis resultados. No eixo dos $y$, representa-se o valor da utilidade, um número abstrato que é agregado a cada um dos possíveis resultados da decisão. Cada resultado tem uma utilidade, sendo que as mais desejáveis têm valores mais altos que as menos desejáveis.

Considerações genéricas sobre o comportamento dos gerentes de exploração de petróleo são ferra- 
Figura 3 - Curvas de comportamento dos gerentes diante do risco

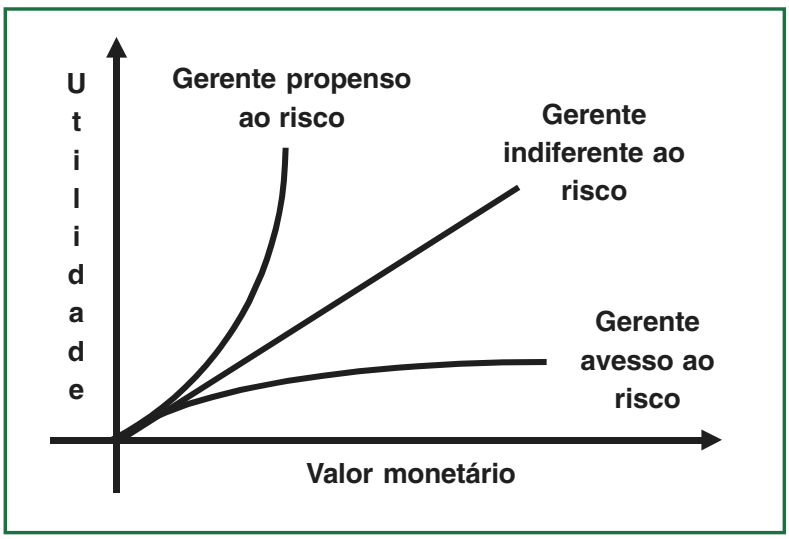

mentas robustas para se delinear um critério de avaliação de prospectos de risco. Assim, a função exponencial do tipo $\mathrm{U}(x)=-\mathrm{e}^{-c x}$ é a mais utilizada na indústria de exploração de petróleo, em que $c$ é o coeficiente de aversão ao risco característico de cada gerente e tem uma forte relação com o tamanho da empresa, ou seja, com o capital exploratório anual da companhia (Walls, 1994).

O coeficiente de aversão ao risco embutido na função utilidade $\mathrm{U}(x)$ é definido pela expressão:

$$
c=-\mathrm{U}^{\prime \prime}(x) / \mathrm{U}^{\prime}(x)
$$

em que U' $(x)$ e U' $(x)$ são a primeira e a segunda derivadas de $\mathrm{U}(x)$, respectivamente.

Esse coeficiente indica como varia o comportamento do investidor em relação ao risco com a magnitude dos valores monetários $(x)$. Assim, $c>0$ indica aversão ao risco, $c=0$ indica indiferença ao risco e $c<0$ indica propensão ao risco.

A aversão ao risco financeiro depende do capital disponível e dos sentimentos do decisor diante do risco. Julgar projetos coerentemente significa avaliar diversos projetos usando o mesmo nível de aversão ao risco. Grande nível de aversão ao risco significa um grande esforço para diminuir as perdas e uma reduzida concentração em grandes ganhos potenciais. Não existe um nível certo ou errado de aversão ao risco. O nosso objetivo é estabelecer o nível de risco que uma firma está preparada para assumir.

Com o intuito de fornecer algum significado para o coeficiente $c$, analistas de decisão utilizam o inverso do coeficiente de aversão ao risco, $1 / c$, como a medida de tolerância ao risco. A unidade de valor da medida de tolerância ao risco toma a unidade da função utilidade de interesse, nesse caso, o valor monetário (US\$, R $\$$, etc.). Mais adi- ante, serão demonstradas algumas maneiras de se calcular o coeficiente de aversão ao risco.

\section{RISCOS}

Os projetos de E\&P de petróleo envolvem geralmente três tipos de riscos: geológicos, econômicos e financeiros.

A probabilidade de sucesso na perfuração de poços de petróleo está associada aos riscos geológicos (existência de um sistema petrolífero), às estimativas de volumes de óleo a serem descobertos e aos tamanhos individuais dos campos de óleo e gás e depende das ferramentas e tecnologias exploratórias utilizadas. Ultimamente, o aumento de informações e o avanço tecnológico, sobretudo em sísmica, modelos geológicos, programas de modelagem e simulação numérica, têm reduzido esses riscos.

Os riscos econômicos estão associados às expectativas futuras do preço do petróleo, aos custos operacionais e dos investimentos, à avaliação acurada dos volumes de óleo a serem produzidos e à realização de seu fluxo de caixa previsto.

Os riscos financeiros estão associados à capacidade de investimentos da firma (capital exploratório), ao número de prospectos disponíveis, às ações políticas, sociais e ambientais que podem embargar o processo exploratório, ao risco de a probabilidade de sucesso estimada estar errada, etc.

A análise quantitativa do risco é fundamental neste trabalho. Aliás, essa metodologia só tem sentido quando se opera com projetos de risco. Se o projeto não tem risco e o tomador de decisão sabe com antecedência tudo o que vai acontecer no futuro, basta fazer uma análise econômica convencional e está tudo resolvido. No entanto, se o projeto envolve riscos, o gerente precisa de uma tecnologia que reduza seus riscos e maximize seus ganhos; nesse caso, a teoria da preferência é importante.

\section{FUNÇÃO UTILIDADE}

Uma forma conveniente de expressar a preferência em relação ao risco é por meio das funções utilidade definidas por Von Neumann e Morgenstern (1953). 
A função utilidade é construída atribuindo-se um valor numérico maior para o melhor resultado possível e um valor numérico menor para o evento menos preferível. A obtenção de valores numéricos intermediários permite construir a curva da função utilidade, que é única para cada tomador de decisão.

\section{NO CENÁRIO INTERNACIONAL, A ATIVIDADE DE EXPLORAÇÃO E PRODUÇÃO DE PETRÓLEO É CONDUZIDA POR ALGUM TIPO DE ASSOCIAÇÃO ENTRE EMPRESAS (JOINT VENTURE), DE MANEIRA A DIMINUIR OS INVESTIMENTOS E OS RISCOS INERENTES A ESSE PROCESSO.}

Para determinar a utilidade que o tomador de decisão agregará a cada possível resultado, deve-se responder a uma série de perguntas que indicarão sua preferência em relação ao risco.

As utilidades requeridas podem ser determinadas em dois passos:

a) Estabelecem-se as utilidades para dois valores monetários arbitrários. A utilidade da melhor consequiência é estabelecida como sendo mais alta do que a utilidade do pior evento. Mostra-se que os resultados finais das análises não dependem dos dois números que foram escolhidos, desde que a utilidade da melhor consequiência seja estabelecida como maior do que a utilidade do pior evento (Nepomuceno e Suslick, 1996).

b) O segundo passo é mais complexo. Nessa etapa, oferece-se ao tomador de decisão uma escolha entre a certeza de um valor monetário e um jogo em que os resultados possíveis são os dois valores monetários cujas utilidades foram estabelecidas arbitrariamente. Para isso, pergunta-se ao decisor se ele prefere a certeza do valor B ou o jogo em que exista a probabilidade $p$ de ganhar A e a probabili- dade $(1-p)$ de perder C. Tentam-se vários valores de $p$ até achar aquele ao qual o decisor é indiferente entre a certeza de B e o jogo. Essa deve ser a utilidade esperada de $\mathrm{B}$, que é igual à utilidade esperada do jogo porque o decisor racional maximiza a utilidade esperada.

Essas curvas, dispostas em um gráfico de valor do investimento versus função utilidade, apresentam-se como uma reta para um tomador de decisão indiferente ao risco, como uma parábola com concavidade para baixo para um tomador de decisão com aversão ao risco e como uma curva convexa para um tomador de decisão amante do risco, conforme mostrado anteriormente na Figura 3.

A função utilidade pode ser definida empírica ou analiticamente. A definição empírica faz-se por meio de uma entrevista, conforme ilustrado anteriormente. Alternativamente, a função utilidade para uma determinada variável pode ser definida utilizando-se expressões analíticas, como nos exemplos seguintes:

a) linear: $\mathrm{U}(x)=c x$

b) exponencial: $\mathrm{U}(x)=-\mathrm{e}^{-c x}$

c) logarítmica: $\mathrm{U}(x)=\ln (x+c), c>0, x>-c$

d) raiz quadrada: $\mathrm{U}(x)=\sqrt{(x+c)}, c>0, x>-c$

Hammond III (1974) mostrou que uma função utilidade exponencial (o único tipo que tem função de aversão ao risco constante) poderia servir como um substituto para muitas outras funções utilidade sob algumas condições. Cozzolino (1980) mostrou que essa função exponencial é importante na avaliação e implementação da análise de risco nos projetos de exploração de petróleo, desde que a maioria das companhias não conheça realmente suas funções utilidade. Walls (1994) demonstrou que a função utilidade mais usada na exploração de petróleo é uma função exponencial do tipo:

$$
\mathrm{U}(x)=-\mathrm{e}^{-c x} \quad(\text { Equação } 2)
$$

em que: $x=$ valor da variável considerada (valor monetário)

$c=$ coeficiente de aversão ao risco $=$ 1/tolerância ao risco

\section{UTILIDADE ESPERADA E EQUIVALENTE CERTO}

Dois conceitos importantes para a aplicação da teoria da preferência foram introduzidos por Pratt (1964). Ele definiu os conceitos de equivalente certo e de função de aversão ao risco associados com a função utilidade. 


\section{Utilidade esperada do projeto}

Utilidade esperada, semelhantemente ao VME, é o somatório das utilidades de cada possível evento multiplicadas pelas suas probabilidades de ocorrência. Um tomador de decisão racional maximizará a utilidade esperada.

Utilidade esperada do projeto é dada por:

$$
\begin{aligned}
& \mathrm{E}(\mathrm{U})=p_{1} \mathrm{U}\left(\mathrm{VPL}_{1}\right)+p_{2} \mathrm{U}\left(\mathrm{VPL}_{2}\right) \\
& \mathrm{E}(\mathrm{U})=-p_{1} \mathrm{e}^{-c \mathrm{VPL}_{1}-p_{2} \mathrm{e}^{-c \mathrm{VPL}_{2}}} \quad(\text { Equação 3) }
\end{aligned}
$$

em que: $c=$ coeficiente de aversão ao risco

$\mathrm{VPL}_{1}=$ valor presente líquido no caso de sucesso

$\mathrm{VPL}_{2}=$ valor presente líquido no caso de insucesso

$p \quad=$ probabilidade de sucesso

\section{Equivalente certo (Eq) e prêmio de risco}

Muitas vezes, uma opção arriscada pode ser comparada a uma opção sem risco. Os executivos poderiam perguntar: qual o menor valor em dinheiro que eu aceitaria, sem risco, como retorno pela escolha arriscada, com esse valor esperado? Esse valor é o equivalente certo da escolha arriscada (Maital, 1996).

Para quem não gosta de risco, ele é geralmente menor do que o valor esperado. A diferença entre o equivalente certo e o valor esperado é o prêmio de risco: o custo em dinheiro da incerteza, como é percebido pela pessoa disposta a tolerá-lo, conforme ilustra a Figura 4.

O equivalente certo (valor ajustado ao risco) é o resultado do desconto do risco baseado na teoria da preferência ao risco, também conhecida como teoria da utilidade.

$\mathrm{O}$ equivalente certo de um projeto de risco é o valor que o decisor está disposto a receber para desistir do projeto (jogo). Exemplo: João participava em um jogo de cara ou coroa em que ele podia ganhar $\mathrm{R} \$ 0$ ou $\mathrm{R} \$ 1.000$ e desistiu quando lhe ofereceram $\mathrm{R} \$ 400$. Assim, para João, o equivalente certo desse jogo é $\mathrm{R} \$ 400$.

Por definição, a utilidade do equivalente certo é igual à utilidade esperada do jogo:

$$
\mathrm{U}(\mathrm{Eq})=\mathrm{E}(\mathrm{u})
$$

Para uma função utilidade do tipo $\mathrm{U}(x)=$ $-\mathrm{e}^{-c x}$, o equivalente certo assumirá a forma expressa na Equação 4.

$$
\underset{i=1}{\mathrm{nq}}=-1 / c \ln \left(\sum p_{i} \mathrm{e}^{-c \mathrm{VPL}_{i}}\right)
$$

em que: $\mathrm{Eq}=$ equivalente certo do projeto $\mathrm{VPL}_{i}=$ valor presente líquido do evento $i$

$$
p_{i} \quad=\text { probabilidade de ocorrência }
$$
do evento $i$

Semelhantemente ao VME, o melhor projeto é aquele que tem o maior equivalente certo.

\section{MAXIMIZAÇÃO DO EQUIVALENTE CERTO}

O decisor racional busca aquela partici-

Figura 4 - Definição do prêmio de risco

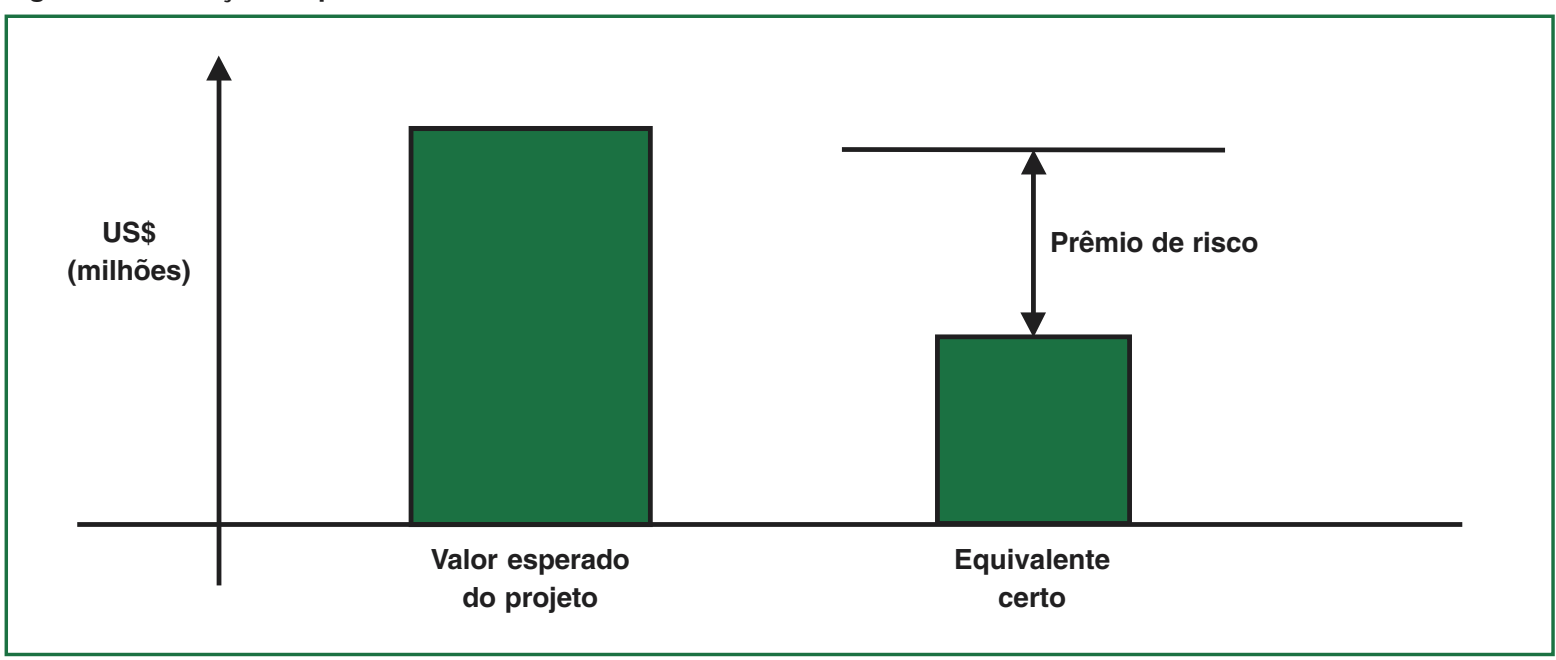


pação que maximiza o valor do equivalente certo.

$$
\mathrm{Eq}(x)=-1 / c \ln \left(p \mathrm{e}^{-c \mathrm{VPL}_{1} x}+(1-\mathrm{p}) \mathrm{e}^{-c \mathrm{VPL}_{2} x}\right)
$$

em que: $x=$ nível de participação no

$$
\text { projeto }(\%)
$$

Tomando-se a primeira derivada e igualando a zero, tem-se:

$$
\frac{d \ln (f(x))}{d x}=\frac{f^{\prime}(x)}{f(x)}=0
$$

Portanto,

$$
X_{\text {ótima }}=\frac{\ln \left[\left(-p V P L_{1} /(1-p) V P L_{2}\right)\right]}{c\left(V P L_{1}-V P L_{2}\right)}
$$

ou, ainda,

(Equação 5)

$$
c=\frac{\ln \left[\left(-p V P L_{1} /(1-p) V P L_{2}\right)\right]}{X_{\text {ótima }}\left(V P L_{1}-V P L_{2}\right)}
$$

\section{(Equação 6)}

Assim, quando, em um projeto $\left(\mathrm{VPL}_{1}\right.$, $\mathrm{VPL}_{2}$, PS), se define um nível de participação desejado, está se determinando também o nível de aversão ao risco. Com isso, os níveis ótimos de participação nos outros projetos da carteira podem ser calculados mantendo-se o mesmo nível de aversão ao risco.

\section{CÁlCULO DO COEFICIENTE DE AVERSÃo AO RISCO}

A seguir, são apresentadas quatro maneiras de calcular o índice de aversão ao risco (IAR):

Método 1: preço de venda do projeto

Método 2: nível de participação desejado no projeto

Método 3: cálculo estatístico da carteira do projeto

Método 4: níveis-padrão das companhias internacionais

Método 1: Coeficiente de aversão ao risco de uma firma e equivalente certo

Esse método, utilizado por Walls (1994), usa um questionário que é submetido ao gerente de exploração para aferir qual seria o preço de venda dos projetos, conforme mostra a Tabela 1.

Tabela 1 - Modelo de questionário sobre o preço de venda dos projetos, a ser respondido pelo gerente de exploração para se calcular o coeficiente de aversão ao risco da firma

\begin{tabular}{|l|c|c|c|c|c|}
\hline Nome & Resultado & $\begin{array}{c}\text { Valor } \\
\text { (US\$ milhões) }\end{array}$ & $\begin{array}{c}\text { Prob. de } \\
\text { sucesso }\end{array}$ & VME & $\begin{array}{c}\text { Preço de } \\
\text { venda }\end{array}$ \\
\hline Proj $_{1}$ & $\begin{array}{c}\text { Sucesso } \\
\text { Insucesso }\end{array}$ & $\begin{array}{c}100 \\
-5\end{array}$ & $10 \%$ & 5,5 & $?$ \\
\hline Proj $_{2}$ & $\begin{array}{c}\text { Sucesso } \\
\text { Insucesso }\end{array}$ & $\begin{array}{c}10 \\
-5\end{array}$ & $40 \%$ & 1,0 & $?$ \\
\hline Proj $_{3}$ & $\begin{array}{c}\text { Sucesso } \\
\text { Insucesso }\end{array}$ & $\begin{array}{c}10 \\
-2\end{array}$ & $50 \%$ & 4,0 & $?$ \\
\hline
\end{tabular}

A relação do valor do equivalente certo $(\mathrm{Eq})$ com o valor monetário esperado (VME) é acompanhada pela medida de aversão ao risco $c$, exibida pela firma (Equação 4).

Exemplo: Considere um caso de apenas um poço, com probabilidade de sucesso de $50 \%$. O valor presente líquido de sucesso é igual a \$10 milhões e o custo do poço seco é de $\$ 2$ milhões.

$\mathrm{VME}=0,5 \times 10+0,5 \times(-2)=\$ 4$ milhões

Como exemplo, assuma que a firma A venderia o poço por $\$ 2$ milhões $\left(\mathrm{Eq}_{\mathrm{A}}\right)$ e a firma $\mathrm{B}$, por $\$ 3$ milhões $\left(\mathrm{Eq}_{\mathrm{B}}\right)$.

O preço pelo qual a firma prefere vender em vez de perfurar representa aquele ponto de indiferença da firma (equivalente certo) entre o valor certo (venda) e um mais lucrativo, porém arriscado (perfurar).

Usando os valores do equivalente certo (\$2 milhões para a firma $\mathrm{A}$ e $\$ 3$ milhões para a firma $\mathrm{B}$ ) e resolvendo a Equação 4, tem-se que a firma A apresenta um nível de aversão ao risco de 0,1203 e a firma B tem um valor de 0,0566.

Método 2: Coeficiente de aversão ao risco de uma firma e nível de participação financeira desejado para um projeto

A aversão ao risco é também expressa pela escolha do nível de participação em um projeto (Equação 5). Assim, apresenta-se um questionário sobre o nível de participação desejado em um projeto para ser respondido pelo gerente de exploração (Tabela 2).

Nesse método (Walls, 1994), o tomador de decisão é questionado para decidir sobre várias oportunidades de investimentos (investimentos em perfuração). O tomador de decisão tem que fazer uma es- 
colha entre seis discretas opções de participação com amplitude variando entre $0 \%$ e $100 \%$. Dessa forma, ele deve escolher o nível de participação que é preferido por sua companhia. Baseado na escolha de participação do tomador de decisão para cada uma das oportunidades de investimento de risco oferecido, um nível de tolerância ao risco da corporação pode ser calculado aproximadamente, utilizando-se uma função exponencial como a forma da função utilidade da firma.

Exemplo: Ofereceram à sua firma perfurar um poço, sendo que os parâmetros são os seguintes: chance de sucesso $=50 \%, \mathrm{VPL}_{1}$ de sucesso $=\$ 13$ milhões, $\mathrm{VPL}_{2}$ de insucesso $=\$-4$ milhões. Dessa forma, escolha sua participação: $100 \%, 75 \%, 50 \%$, $25 \%, 12,5 \%, 0 \%$.

A aversão ao risco pode ser expressa pela escolha do nível de participação em um projeto, conforme mostrado na Equação 6.

Assim, se sua seleção for:

$100 \%$, seu nível de aversão ao risco será de 0,000693 ; $75 \%$, seu nível de aversão ao risco será de 0,000924; $50 \%$, seu nível de aversão ao risco será de 0,001387 ; $25 \%$, seu nível de aversão ao risco será de 0,002773 ; $12,5 \%$, seu nível de aversão ao risco será de 0,005547 ; $0 \%$, seu nível de aversão ao risco será de $\gg 1$.

Quando um gerente especifica o nível de participação desejado em um projeto, ele define o coeficiente de aversão ao risco apresentado pela firma. Por meio da aplicação do mesmo nível de aversão ao risco em todos os projetos, é dada coerência ao processo de tomada de decisão, preservando a firma contra perdas financeiras que ela não seria capaz de suportar.

\section{Método 3: Análise de decisões anteriores}

Nepomuceno (1997) introduziu um método para facilitar a determinação do coeficiente de aversão ao risco pelo gerente de exploração, expondo em um gráfico toda a carteira de projetos da firma versus o nível de tolerância ao risco requerido por cada projeto para que a participação ótima seja de $100 \%$ (Figura 5).

A partir da Figura 5, o gerente de exploração poderá definir de quais projetos, dentre os 21 disponíveis na carteira, ele gostaria de participar com $100 \%$. Quando esses projetos estão estabelecidos, o nível de participação ótima em cada projeto é determinado automaticamente, utilizando-se a Equação 6.

\section{SE O PROJETO NÃO TEM RISCO \\ E O TOMADOR DE DECISÃO \\ SABE COM ANTECEDÊNCIA \\ TUDO O QUE VAI ACONTECER \\ NO FUTURO, BASTA FAZER UMA \\ ANÁLISE ECONÔMICA \\ CONVENCIONAL E ESTÁ \\ TUDO RESOLVIDO.}

\section{Método 4: Níveis-padrão das companhias internacionais}

Um estudo empírico de Walls e Dyer (1992), no qual foi utilizado o modelo de teoria da preferência, mediu o coeficiente de aversão ao risco aplicado nas 18 maiores, independentes e integradas companhias de petróleo no período de 1981 a 1990, descobrindo uma regra simples que relaciona a tolerância ao risco da firma com o seu nível de orçamento anual. Essa relação indica que, como primeira aproximação, o valor da tolerância ao risco de uma firma é igual a 1/4 do seu orçamento anual da exploração. Por

Tabela 2 - Modelo de questionário sobre nível de participação no projeto, a ser respondido pelo gerente de exploração para se calcular o coeficiente de aversão ao risco da firma

\begin{tabular}{|c|c|c|c|c|c|c|c|c|c|}
\hline Nome & Resultado & $\begin{array}{c}\text { Valor } \\
\text { (US\$ milhões) }\end{array}$ & $\begin{array}{l}\text { Prob. de } \\
\text { sucesso }\end{array}$ & \multicolumn{6}{|c|}{$\begin{array}{c}\text { Nível de participação } \\
\text { desejado }\end{array}$} \\
\hline Proj $_{1}$ & $\begin{array}{l}\text { Sucesso } \\
\text { Insucesso }\end{array}$ & $\begin{array}{r}100 \\
-5\end{array}$ & $10 \%$ & $100 \%$ & $75 \%$ & $50 \%$ & $25 \%$ & $12,5 \%$ & $0 \%$ \\
\hline Proj $_{2}$ & $\begin{array}{l}\text { Sucesso } \\
\text { Insucesso }\end{array}$ & $\begin{array}{l}10 \\
-5\end{array}$ & $40 \%$ & $100 \%$ & $75 \%$ & $50 \%$ & $25 \%$ & $12,5 \%$ & $0 \%$ \\
\hline Proj $_{3}$ & $\begin{array}{l}\text { Sucesso } \\
\text { Insucesso }\end{array}$ & $\begin{array}{l}13 \\
-4\end{array}$ & $50 \%$ & $100 \%$ & $75 \%$ & $50 \%$ & $25 \%$ & $12,5 \%$ & $0 \%$ \\
\hline
\end{tabular}


exemplo, uma firma com um orçamento de exploração anual de 40 milhões teria um nível de tolerância ao risco aproximadamente igual a $0,10 \times 10^{-6}$. Entretanto, devese notar que essa regra representa somente um ponto para o início da avaliação do

\section{SE O PROJETO ENVOLVE RISCOS, O GERENTE PRECISA \\ DE UMA TECNOLOGIA QUE REDUZA SEUS RISCOS E MAXIMIZE SEUS GANHOS; NESSE CASO, A TEORIA DA PREFERÊNCIA É IMPORTANTE.}

coeficiente de aversão ao risco da firma individual. Seria fácil imaginar duas firmas com orçamentos anuais de exploração idênticos com níveis de aversão ao risco significativamente diferentes. Essas diferenças são motivadas por diversos fatores, destacando-se a filosofia gerencial da exploração, a política de risco da corporação, a contribuição da unidade de negócio de exploração para o portifólio da corporação, etc.

\section{APLICAÇÃO PRÁTICA EM UM MODELO}

\section{O problema}

Assuma que a exploração de petróleo no país seja feita de acordo com os termos de um contrato de risco. No cenário internacional, a atividade de exploração e produção de petróleo é conduzida por algum tipo de associação entre empresas (joint venture), de maneira a diminuir os investimentos e os riscos inerentes a esse processo.

O modelo agregado simplificado, utilizado nesse trabalho de alocação de recursos, está ilustrado na Figura 6.

O país tem três bacias cujas perspectivas geológicas são de descobertas de grandes campos na bacia A (tamanho médio dos campos de 100 milhões de barris), de campos médios na bacia B (média de 20 milhões de barris) e de campos pequenos na bacia $\mathrm{C}$ (média de 10 milhões de barris).

A distribuição do tamanho de campos de petróleo tem a forma de uma curva log-normal e é possível usar essa curva para a análise do problema. No entanto, para simplificar o exemplo, usa-se o tamanho médio desses campos para a avaliação econômica dos projetos (Tabela 3 ).

Os dados de cada bacia podem ser sintetizados conforme mostra a Tabela 3.

Na Tabela 3, FAE é o Fator de Aproveitamento Econômico do campo definido por Silva e Terrana (1989), dado pela relação: FAE = VPL/Volume $x$ Preço.

Figura 5 - Tolerância ao risco dos projetos da carteira de projetos para uma participação ótima de $100 \%$

\section{Tolerância ao Risco (TR) requerida pelos projetos para que a participação ótima seja de $100 \%$}

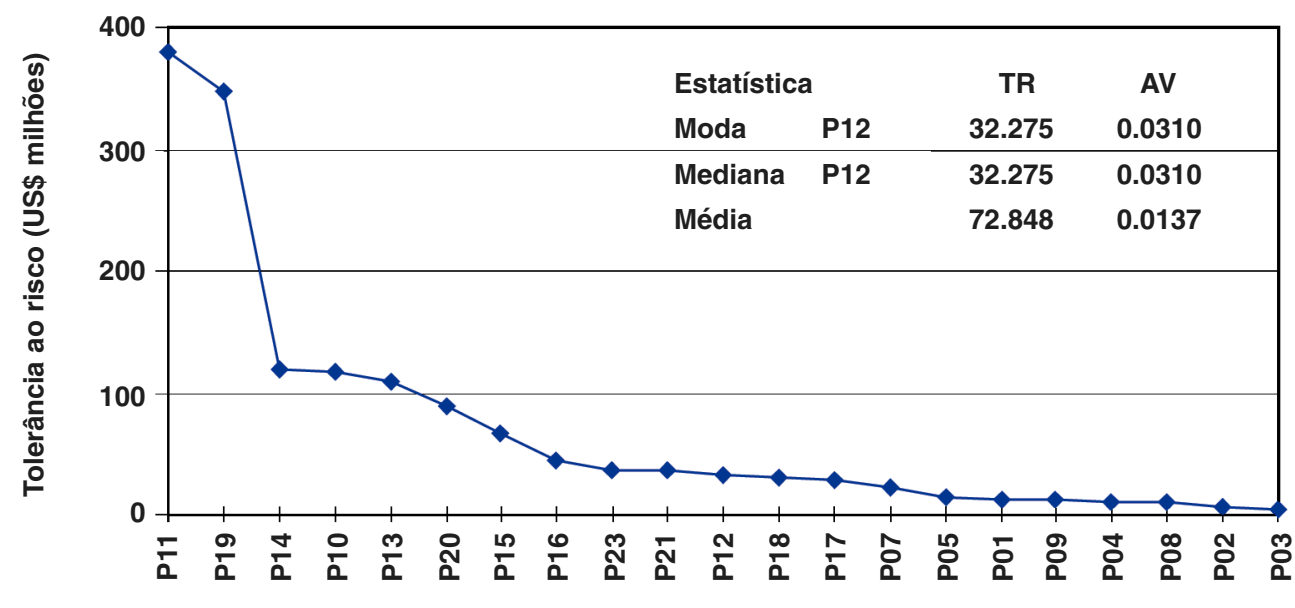

Carteira de projetos 
Ou seja,

VPL = FAE $x$ Volume $x$ Preço

(Equação 7)

Tabela 3 - Sumário dos dados do modelo

\begin{tabular}{|l|c|c|c|}
\hline & Bacia A & Bacia B & Bacia C \\
\hline $\begin{array}{l}\text { Tamanho médio dos } \\
\text { campos (milhões de } \\
\text { barris) }\end{array}$ & 100 & 20 & 10 \\
\hline $\begin{array}{l}\text { Custos dos projetos } \\
\text { (US\$ milhões) }\end{array}$ & 8 & 6 & 3 \\
\hline $\begin{array}{l}\text { Probabilidade de } \\
\text { sucesso (\%) }\end{array}$ & 25 & 25 & 25 \\
\hline Preço do óleo (US\$) & 18 & 18 & 18 \\
\hline FAE (\%) & 30 & 30 & 30 \\
\hline
\end{tabular}

\section{Solução do problema}

Conforme visto ao longo do trabalho, os pontos críticos da aplicação da teoria da preferência estão na determinação da função utilidade e do coeficiente de aversão ao risco do gerente diante da capacidade financeira da firma.

Neste artigo, utiliza-se a função exponencial do tipo $\mathrm{U}(x)=-\mathrm{e}^{-c x}$, que, como referido anteriormente, é a função utilidade mais usada na exploração de petróleo e que apresenta uma série de vantagens, como, por exemplo, ter o coeficiente de aversão ao risco como uma constante, restringindo nosso trabalho à determinação desse coeficiente.

A Equação 5 define a relação entre o nível de participação no projeto e o índice

Figura 6 - Agrupamento das diversas bacias sedimentares de um país em três grandes áreas (três grandes bacias) para a construção de um modelo simplificado

MODELO ADOTADO PARA QUALIFICAÇão do ORÇAMENTO NA ÁREA DE EXPLORAÇÃo DE PETRÓLEO

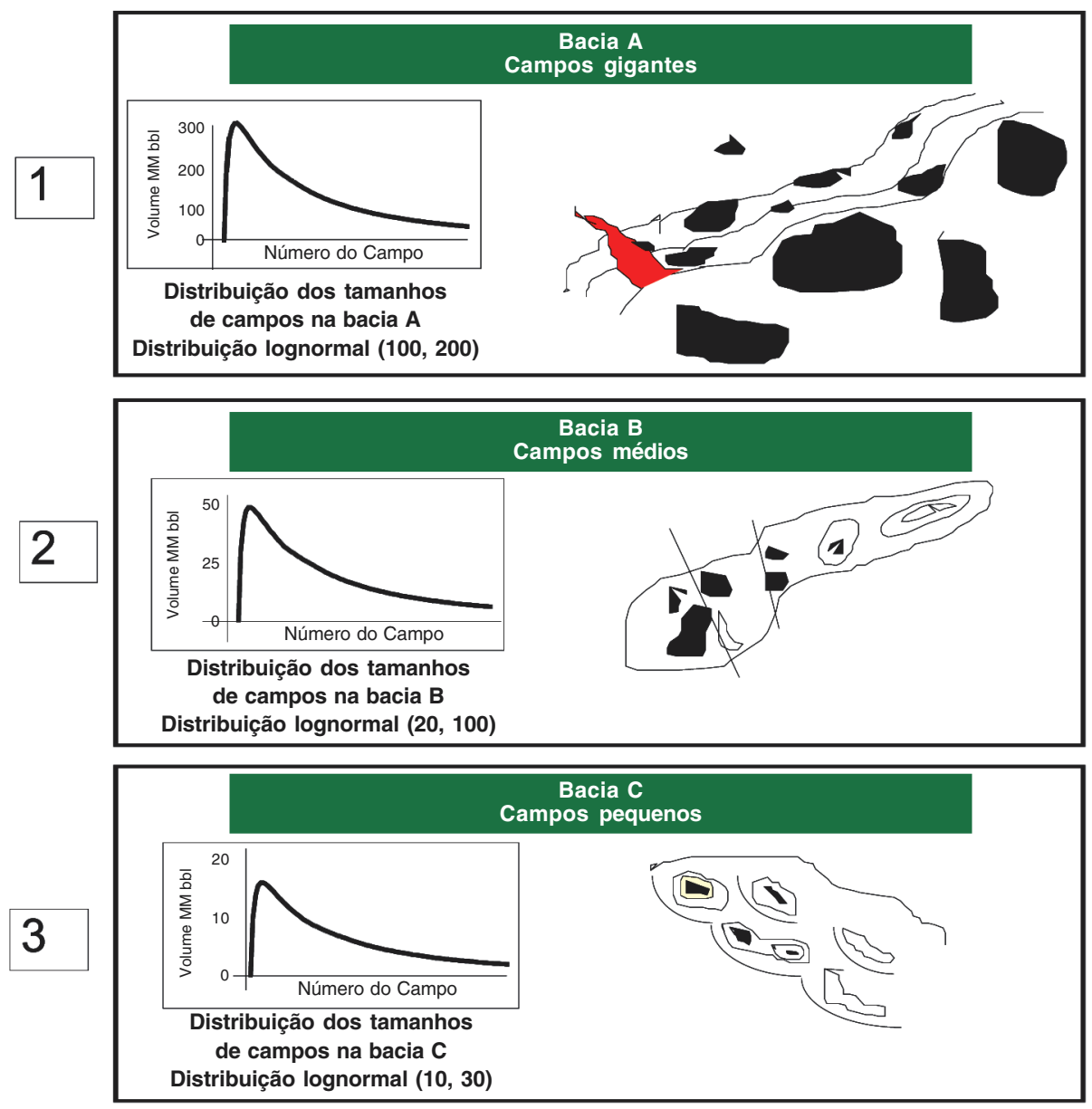


de aversão ao risco. Assim, com a ajuda da Figura 7, construída a partir dessa equação, será feita a seguinte análise, visando a definir o coeficiente de aversão ao risco da firma:

1. Para uma situação de monopólio (100\% de participação em todos os projetos, independentemente do risco dos projetos), o nível de aversão ao risco varia de projeto para projeto, tornando-se inconsistente com a função de preferência definida anteriormente, $\mathrm{U}(x)=-\mathrm{e}^{-c x}$, que estabelece um coeficiente de aversão ao risco como uma constante $(c)$ que será aplicada a todos os projetos, independentemente do valor do projeto $(x)$.

2. Adotando-se o nível de aversão igual a $c_{3}$, valor que define um nível ótimo de $100 \%$ para os projetos da bacia $\mathrm{C}$, a participação nos projetos da bacia B será ótima para $52 \%$ e, para os projetos da bacia A, será de $18 \%$. Esse dado pode ser extraído diretamente da Figura 7 ou calculado analiticamente pela fórmula da Equação 5. As Figuras 8 e $9^{3}$ ilustram o nível de participação ótimo para cada bacia, quando se usa um coeficiente de aversão ao risco $c_{3}=0,0314$. Esse é um dado surpreendente; o nível ótimo de participação nos melhores projetos não é, necessariamente, $100 \%$, como se pode pensar intuitivamente.
3. O gráfico mostra claramente que, para uma participação de $100 \%$, a ordem de importância dos projetos é: C > B > A. ${ }^{4}$ Por outro lado, para um nível de participação financeira inferior a $60 \%$, tem-se uma ordem inversa: A > B > C. Isso mostra a importância da associação tipo joint venture (participação fracionada) nos projetos de risco.

\section{A TEORIA DA PREFERÊNCIA ESPECIFICA O NÍVEL ÓTIMO DE PARTICIPAÇÃO FINANCEIRA EM UM PROJETO, CONSIDERANDO AS RESTRIÇÕES DE CAPITAL DA FIRMA E A DISPOSIÇÃO DE SEUS GERENTES DE CORRER RISCOS.}

Para definir o nível ótimo de alocação de capital exploratório para cada bacia, será considerado o nível de aversão ao risco como o inverso da tolerância ao risco, que é igual a 1/4 do capital exploratório anual da firma conforme discutido anteriormente.

Figura 7 - Coeficiente de aversão ao risco versus nível de participação financeira nos projetos médios das bacias A, B e C

Nível de participação ótima versus

coeficiente de aversão ao risco

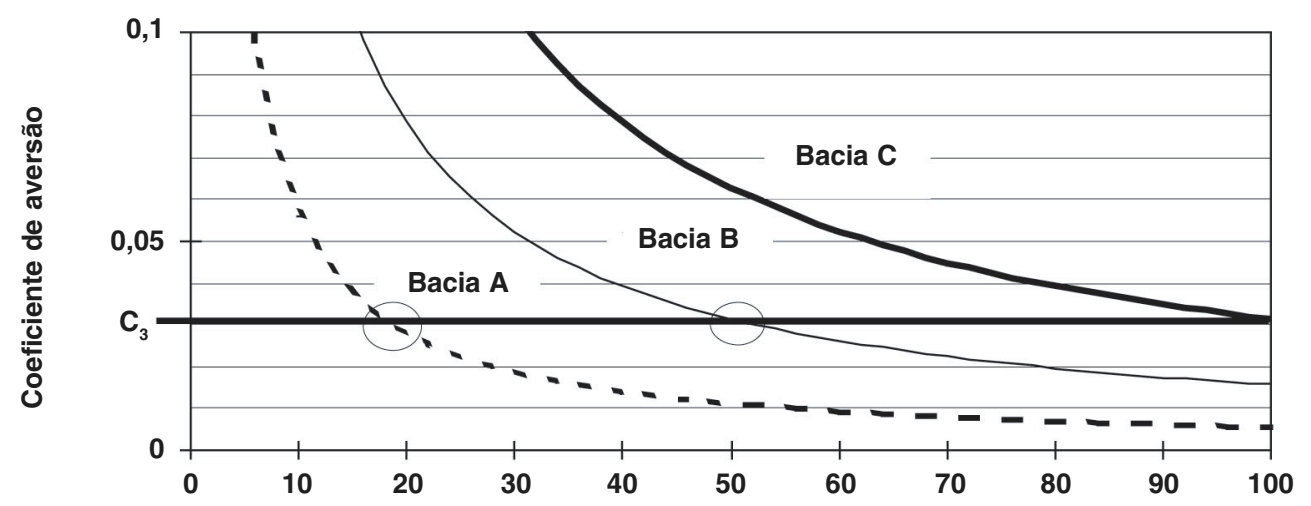

Nível de participação no projeto (\%) 
Assim, é possível expressar a alocação ótima de recursos em exploração com o auxílio da Figura 9, ou seja, definindo-se qual o nível de participação desejado em cada projeto de uma bacia, determinase qual é o capital exploratório anual ideal para essa bacia. Reciprocamente, dado o capital exploratório de uma firma, é possível definir o nível ótimo de participação em cada uma das bacias aplicando diretamente o gráfico da Figura 9. Assim, para uma capital exploratório de US\$ 127 milhões, os níveis ótimos de participação em cada bacia são: $18 \%$ na bacia $\mathrm{A}, 50 \%$ na bacia $\mathrm{B}$ e $100 \%$ de participação nos projetos da bacia $\mathrm{C}$.

O capital ótimo total da joint venture para cada uma dessas três bacias é: Ka $=$ US $\$ 704$ milhões, $\mathrm{Kb}=\mathrm{US} \$ 254$ milhões e Kc $=$ US\$ 127 milhões. Como referido nos itens anteriores, esse método aloca mais dinheiro nas melhores bacias, mas isso não significa que o nível de participação de uma firma nos projetos da melhor bacia deva necessariamente ser maior.

Figura 8 - Equivalente certo versus nível de participação financeira nos projetos médios das bacias A, B e C

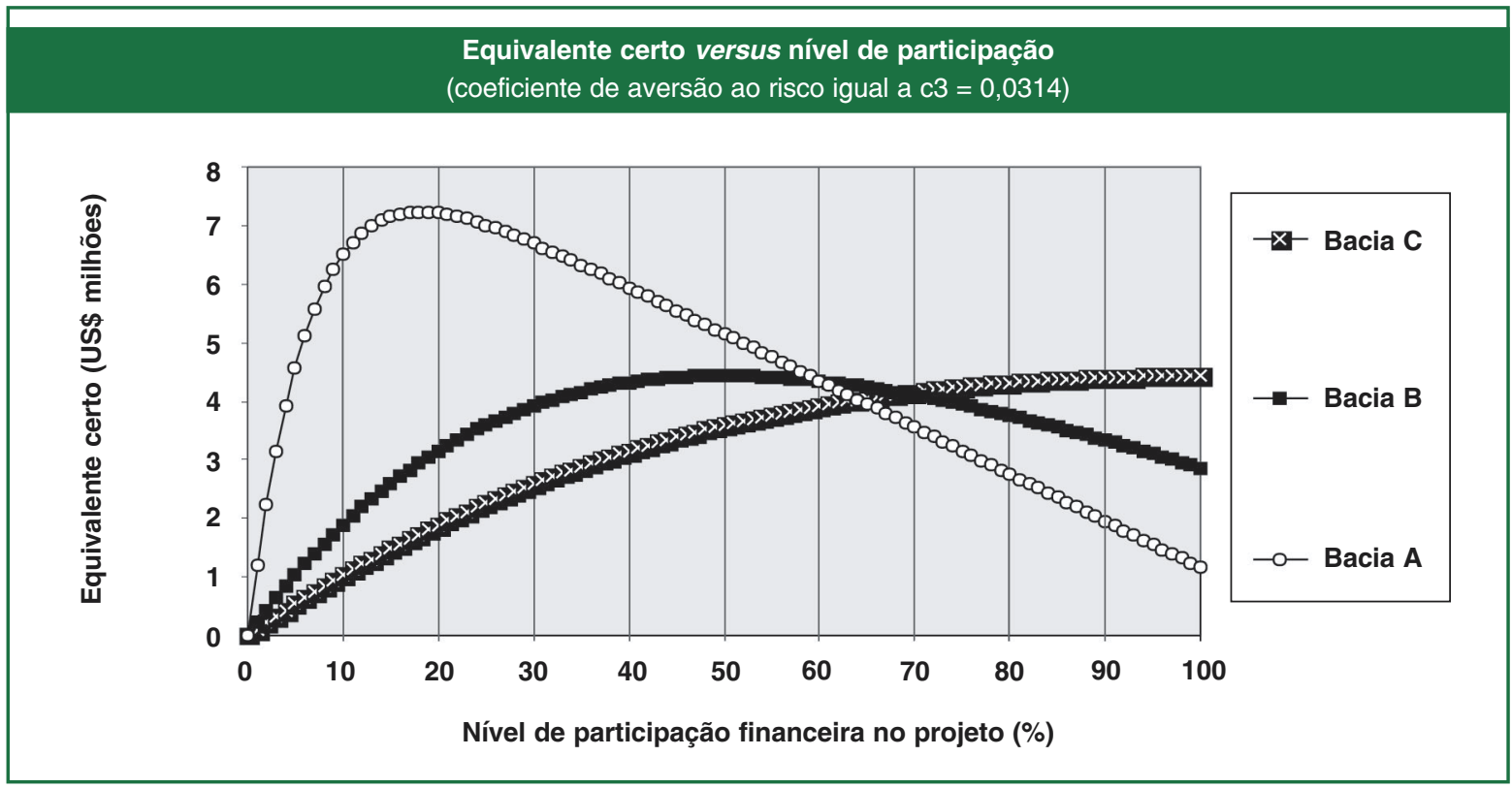

Figura 9 - Nível de participação desejada em cada projeto das bacias A, B e C versus alocação de capital exploratório ideal para a firma

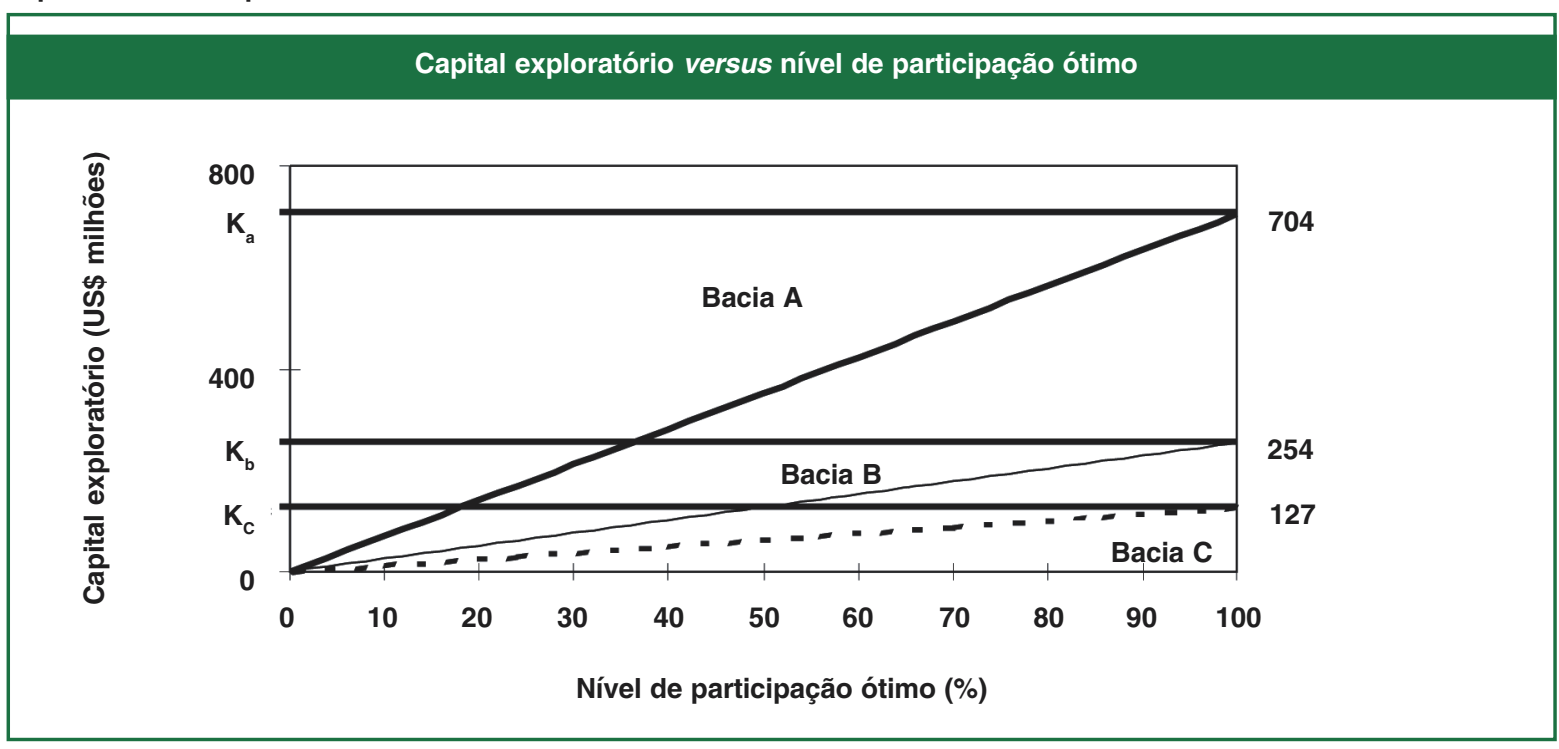




\section{CONSIDERAÇÕES FINAIS}

A metodologia baseada na teoria da preferência, apresentada neste trabalho, permite criar um processo coerente de diversificação e compartilhamento de investimentos na exploração de petróleo, definindo o nível ótimo de participação financeira para projetos desenvolvidos em parcerias, visando à maximização do ganho e à redução do risco e respeitando a capacidade financeira da firma e a disposição de seus gerentes de correr riscos.

Um outro aspecto importante dessa metodologia é a possibilidade de fornecer melhor qualidade às decisões, permitindo a análise dessas decisões a posteriori.

Além da sua aplicação na exploração de petróleo, essa metodologia para tomada de decisão em projetos de risco é muito ampla, indo da escolha de uma carteira de ações na bolsa de valores até decisões pes- soais como a opção por um determinado emprego.

As grandes dificuldades para a implantação dessa metodologia são: encontrar a função utilidade da firma para cada um dos objetivos e determinar o nível de aversão ao risco apresentado pela firma. Mostra-se neste artigo que o questionário específico da indústria, a reanálise das decisões de alocações passadas e a análise conjunta de todos os projetos disponíveis na carteira fornecem uma medida útil para estimar o coeficiente de aversão ao risco da firma.

A aplicação prática ilustrada neste trabalho mostra claramente a importância da participação fracionada em projetos de risco nas associações do tipo joint venture. O exemplo mostrou que, para uma participação de $100 \%$, a ordem de importância dos projetos é: C é melhor do que B e B é melhor do que A. Por outro lado, para uma participação inferior a $60 \%$, tem-se uma inversão de prioridades, ou seja, o projeto A é melhor do que o projeto $B$, que, por sua vez, é melhor do que o projeto $\mathrm{C}$. $\mathrm{O}$

REFERÊNCIAS BIBLIOGRÁFICAS

COZZOLINO, John M. Controlling risk in capital budgeting: a practical use of utility theory for measurement and control of petroleum exploration risk. The Engineering Economist, v. 25 , n. 3, p. $161-86,1980$.

HAMMOND III, J. S. Simplifying the choice between uncertain prospects where preference is nonlinear. Management Science, v. 20, $\mathrm{n}$. 7, p. 1047-72, Mar. 1974.

MAITAL, S. Economia para executivos. Rio de Janeiro: Campus, 1996.
NEPOMUCENO, F. Tomada de decisão em projetos de risco na exploração de petróleo. Campinas: Unicamp/IG/DARM, 1997. 243p. (Tese de Doutorado).

NEPOMUCENO, F., SUSLICK, S. B. USo da teoria da preferência em projetos de exploração de petróleo. XXXIX Congresso Brasileiro de Geologia, v. 7, p. 136-40 Salvador: set. 1996.

PRATT, J. W. Risk aversion in the small and in the large. Econometrica, v. 32, n. 1, p. 12236, Jan. 1964.

SILVA, R. R., TERRANA, A. C. Orientação para o uso do SIPLEX89. Rio de Janeiro: Petrobrás, 1989. (Relatório interno).
Von NEUMANN, J., MORGENSTERN, 0. Theory of games and economic behavior. 3. ed. Princeton: Princeton University Press, 1953.

WALLS, M. R. Corporate risk tolerance and capital allocation: a practical approach to setting and implementing an exploration risk policy. In: Managing risk and strategic decisions in petroleum exploration. Rio de Janeiro: Petrobras, dez. 1994. (Seminar).

WALLS, M. R., DYER, J. S. Risky propensity and firm performance: a study of the petroleum exploration industry. Colorado: Colorado School of Mines, 1992. (Working Paper Series n. 92-9).

\section{NOTAS}

Os autores gostariam de agradecer 0 apoio do CNPq para a realização desta pesquisa.

1. Na indústria de petróleo, o conceito de prospecto refere-se a áreas que contêm indícios ou possibilidades de acumulação de óleo e/ou gás que possam ser extraídos economicamente no futuro.

\footnotetext{
2. Exemplo: em um jogo de moeda (cara ou coroa), com $50 \%$ de chance de ganhar 10 reais ou $50 \%$ de chance de perder 5 reais, certamente haverá muitos jogadores. Mas, se mudarmos os valores para ganhar 100 mil reais ou perder 50 mil reais, haverá poucos jogadores, evidenciando uma aversão ao risco dos indivíduos quando os valores são elevados comparativamente ao seu capital, muito embora - VME seja 10 mil vezes maior que na situação anterior.
}

3. Comparação de perfis de risco dos projetos médios das três regiões considerando como índice de aversão ao risco 0 valor $C_{3}$, que é 0 valor que maximiza o equivalente certo do projeto médio da bacia $\mathrm{C}$ para uma participação de $100 \%$

4. 0 símbolo "A > B" significa que os projetos da bacia "A são melhores do que os da B". 